\title{
Angular domain fluorescence imaging for small animal research
}

\section{Fartash Vasefi}

Simon Fraser University

The School of Engineering Science

8888 University Drive

Burnaby, British Columbia, Canada V5A 1S6 and

Lawson Health Research Institute

Imaging Program

268 Grosvenor St.

London, Ontario, Canada N6A 4V2

\section{Michelle Belton}

Lawson Health Research Institute Imaging Program, 268 Grosvenor St. London, Ontario, Canada N6A 4V2

\section{Bozena Kaminska}

Simon Fraser University

The School of Engineering Science

8888 University Drive

Burnaby, British Columbia, Canada V5A 1S6

\section{Glenn H. Chapman}

Simon Fraser University

The School of Engineering Science

8888 University Drive

Burnaby, British Columbia, Canada V5A 1S6

\section{Jeffrey J. L. Carson}

Lawson Health Research Institute

Imaging Program

268 Grosvenor St.

London, Ontario, Canada N6A 4V2 and

University of Western Ontario

Medical Biophysics

Room 407 Medical Sciences Building

London, Ontario, Canada N6A 5C1

\begin{abstract}
We describe a novel macroscopic fluorescent imaging technique called angular domain fluorescence imaging (ADFI) applicable to the detection of fluorophores embedded in biological tissues. The method exploits the collimation detection capabilities of an angular filter array (AFA). The AFA uses the principle of acceptance angle filtration to extract minimally scattered photons emitted from fluorophores deep within tissue. Our goal was to develop an ADFI system for imaging near-infrared fluorescent markers for small animal imaging. According to the experimental results, the ADFI system offered higher resolution and contrast compared to a conventional lens and lens-pinhole fluorescent detection system. Furthermore, ADFI of a hairless mouse injected with a fluorescent bone marker revealed vertebral structural and morphometric data that correlated well with data derived from volumetric x-ray computed tomography images. The results suggested that ADFI is a useful technique for submillimeter mapping of the distribution of fluorescent biomarkers in small animals. (C) 2010 Society of Photo-Optical Instrumentation Engineers. [DOI: 10.1117/1.3281670]
\end{abstract}

Keywords: optical imaging; scattering; angular filters; fluorescence imaging; macroscopic imaging; small animal imaging; BoneTag.

Paper 09219LRR received Jun. 3, 2009; revised manuscript received Nov. 6, 2009; accepted for publication Nov. 11, 2009; published online Jan. 20, 2010.
Fluorescent agents are important tools for mechanistic studies on biomolecular function and disease processes. ${ }^{1,2}$ Confocal and multiphoton microscopies have emerged as powerful methods for imaging of fluorescent agents in tissues up to a few hundred micrometers below the surface of skin. ${ }^{3}$ Alternative methodologies based on macroscopic epiillumination imaging have employed sensitive cameras to acquire surface-weighted images of fluorescence from agents up to a few millimeters below the surface of the skin. ${ }^{4}$ These imaging approaches have been highly successful in furthering our understanding of biological systems, but they have been limited to imaging function and processes near the surface

Address all correspondence to: Jeffrey J. L. Carson, Lawson Health Research Institute, Imaging Program, 268 Grosvenor St., London, Ontario, Canada N6A 4V2. Tel: 519-646-6100 x.64767. Fax: 519-646-6110; E-mail: jcarson@lawsonimaging.ca. largely on account of the optical scattering properties of tissue. Scatter degrades the spatial resolution as the depth increases. Therefore, there is a need for new methodologies that can overcome the limitations imposed by optical scatter to enable the capture to in vivo fluorescence images with larger fields of view and precise measures of fluorescent agent distribution at deeper sites within tissues.

Here, we describe the use of a silicon micromachined angular filter array (AFA) to reject unwanted scattered photons and pass the image-forming photons (in the form of ballistic and quasi-ballistic photons from the source) that have straight or nearly straight trajectories. ${ }^{5-9}$ The angular filter array discriminates nondeviated photons from the much more numerous diffusely scattered photons emitted from a fluorophore embedded at depth within tissue. As shown in Fig. 1(a), the

1083-3668/2010/15(1)/016023/5/\$25.00 @ 2010 SPIE 

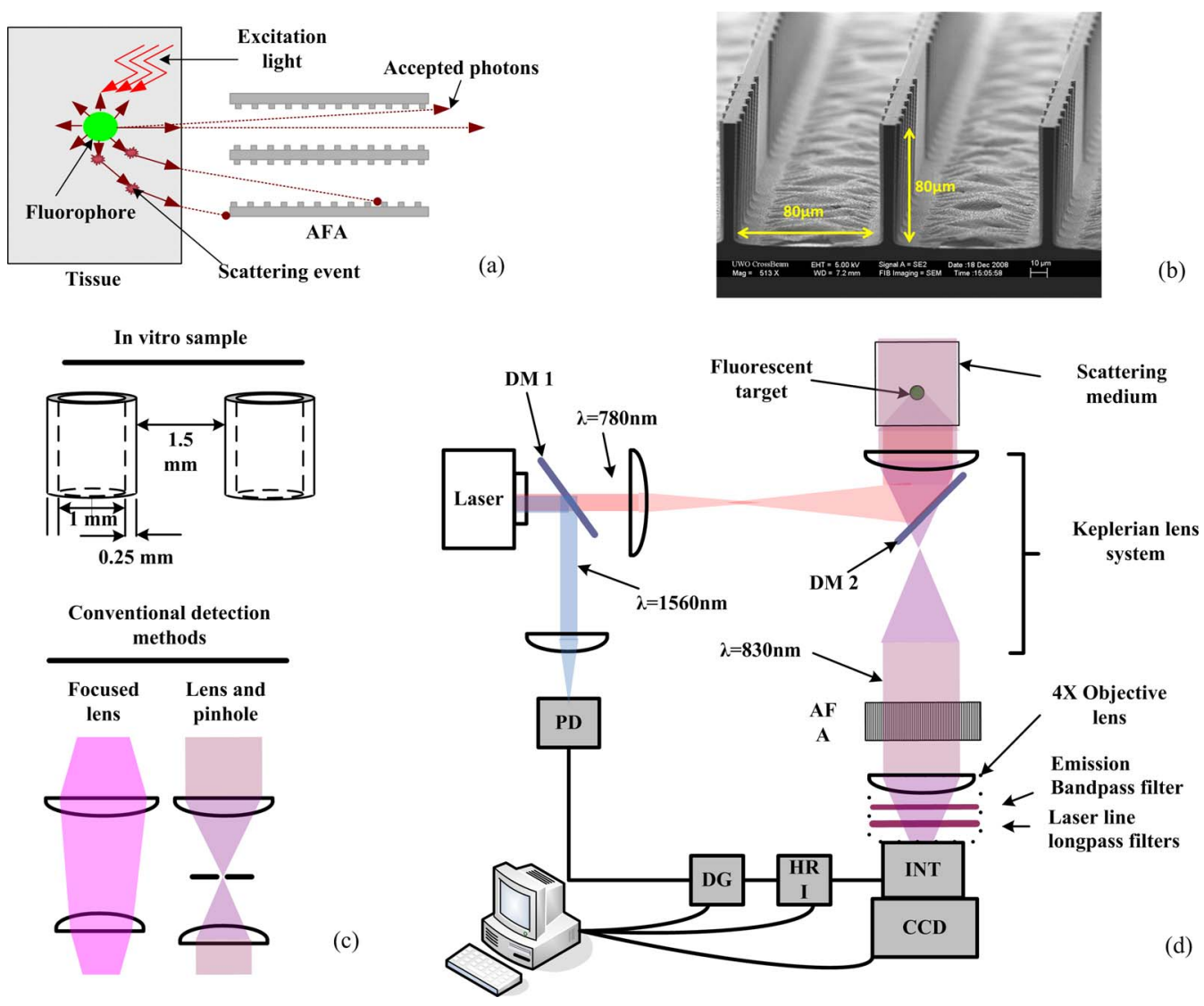

Fig. 1 Principle and method for angular domain fluorescent imaging. (a) Angular domain fluorescent imaging principle. (b) Silicon micromachined angular filter array (bottom piece). (c) Diagram of glass capillary tubes used to contain fluorescent agents during phantom-based experiments (top). Schematic of imaging setups used to compare performance of ADFI system (bottom). (d) Optical imaging setup (CCD, charge-coupled device; HRI, high-rate imager; INT, intensifier; DG, delay generator; DM, dichroic mirror; AFA, angular filter array; and PD, photodiode).

multiply scattered photons emitted from the fluorophore are less likely to exit from the tissue surface with a trajectory acceptable to the AFA, while those that are not scattered (or weakly scattered) will have a trajectory within the angular acceptance range of the AFA. Consequently, the accepted photons will be detected by the camera, whereas the multiply scattered photons will not. The resultant line image formed on the camera represents a projection of the fluorophore distribution within the tissue. The performance of the AFA is not dependent on coherence or the wavelength of light. ${ }^{6}$ We tested the performance of angular domain fluorescence imaging (ADFI) to map fluorophores over a large field of view with submillimeter spatial resolution at tissue depths up to $2 \mathrm{~mm}$.

The main component of the AFA was an array of 1.5-cm-long microtunnels micromachined on to a silicon substrate, as shown in Fig. 1(b). The opening of each microtunnel was square in cross section and had dimensions of $80 \mu \mathrm{m}$ $\times 80 \mu \mathrm{m}$ (acceptance angle $\sim 0.42 \mathrm{deg}$ ). The walls of the AFA were patterned with many small features $(2 \mu \mathrm{m}$ height with periodicity of $20 \mu \mathrm{m}$ ) to suppress internal reflections within each microtunnel. A flat silicon wafer was used as the top piece to enclose the microtunnels to form the AFA (not shown). The aspect ratio and microtunnel size for the square geometry provided optimal image contrast compared to other filter geometries. ${ }^{10}$ An alternative to the AFA is the lenspinhole optical setup that provides similar angular filtration to the AFA and can be realized by inserting a pinhole into a Keplerian lens system [Fig. 1(c)]. However, our previous work has shown that, in comparison to the pinhole, the AFA has superior spatial resolution ${ }^{7}$ and is insensitive to lens aberrations.

The 1-D linear array of microtunnels necessitated a scanning system for the capture of 2-D images. We employed a computer-controlled $z$-axis stage to incrementally raise the sample between scans. One horizontal line image of the sample was taken through the AFA at each step, and a final 2-D image was assembled from the stacked line images. Hence, an entire region of the sample could be passed through the field of view of the AFA and imaged. The experimental setup shown in Fig. 1(d) was based on the reflection-based angular domain imaging configuration. A thin uniform line of light from a pulsed laser source was used to excite the fluorescent target. The AFA accepted the minimally deviated photons, while the photons outside the acceptance angle of the AFA were rejected. The addition of time gating to the imaging system created a mode-lock mechanism that minimized the effects of stray light by synchronization of the gate intensified CCD with the incoming laser pulse. Although we used a pulsed illumination setup, the gate width of the camera was wide enough to capture the entire light pulse mimicking a CW laser setup (i.e., early arriving photons were not discriminated). The light intensity on the detection side of the AFA 


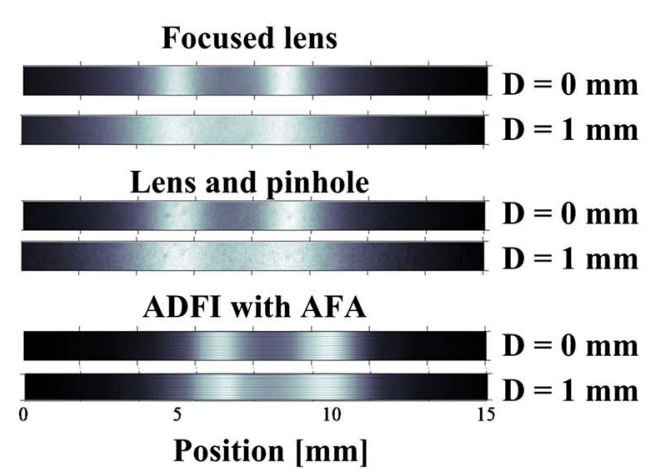

(a)

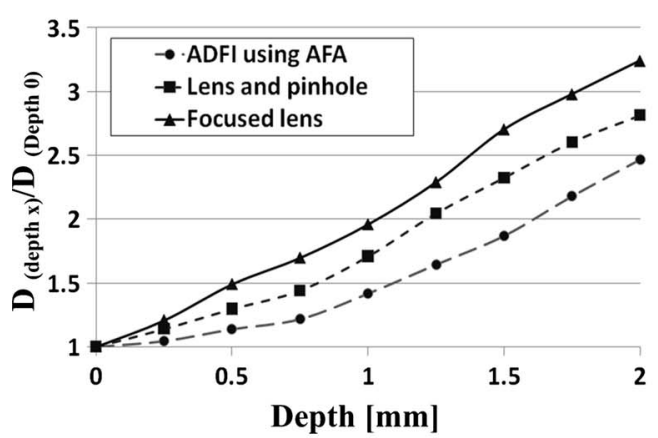

(c)

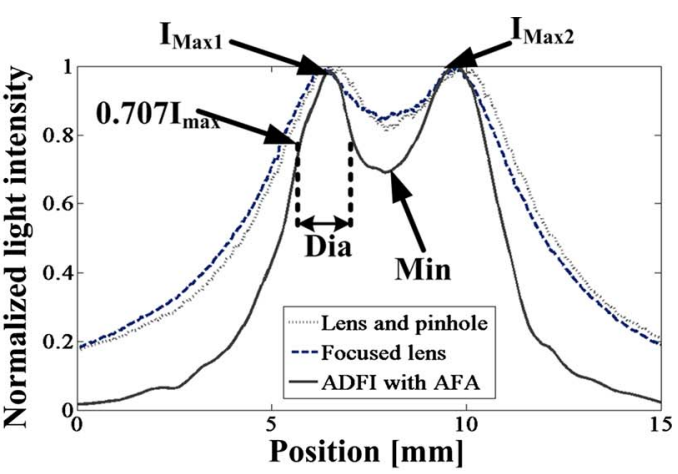

(b)

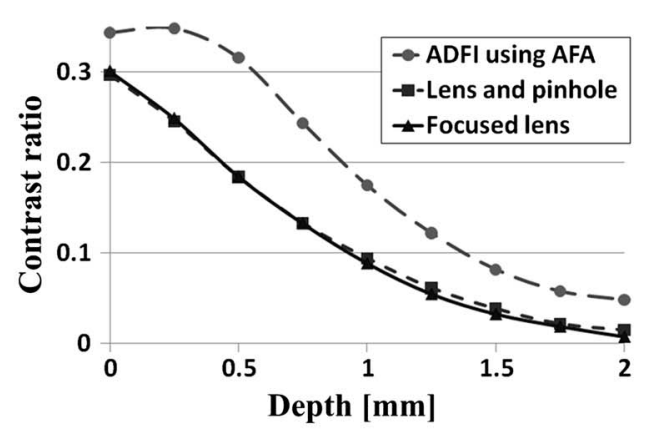

(d)

Fig. 2 Angular domain fluorescent imaging in turbid media. Targets were two glass tubes filled with $20 \mu \mathrm{M}$ DTTC diluted in ethanol and placed at various depths $(D \leqslant 2 \mathrm{~mm})$ from the detection surface of an optical cuvette $(5 \mathrm{~cm} \times 5 \mathrm{~cm} \times 2 \mathrm{~cm})$ filled with $1 \%$ Intralipid: (a) image from lens system focused ( $f / 2$ ) to the surface of the sample (top), lens and pinhole system (acceptance angle $=1.15$ deg) (middle), and ADFI using AFA (bottom). (b) Line profiles from the images in (a) for $D=1 \mathrm{~mm}$. (c) Spatial resolution analysis of the fluorescent targets as a function of depth. (d) Contrast ratio analysis of the fluorescent targets as a function of depth.

had a sinusoidal pattern due to the periodic pattern of microchannels and walls between them. To remove the artifact effect of the microchannel profile on the resultant images (as shown in our previous work ${ }^{5-10}$ ), the upper limit envelope of the sinusoidal pattern was calculated for each AFA line profile to interpolate the maximum light intensity at each microchannel.

In order to evaluate the performance of ADFI, the spatial resolution and contrast were quantified and compared with results from two conventional fluorescent imaging configurations (a focused lens system and a Keplerian lens and pinhole system), as shown in Fig. 1(c). Spatial resolution and contrast were quantified by fluorescent imaging of two glass tubes filled with 3,3'-diethylthiatricarbocyanine iodide (DTTC, $20 \mu \mathrm{M})$ embedded in a $1 \%$ Intralipid scattering medium. Figure 2(a) presents representative fluorescent images for the three detection methods at two depths within the scattering medium (i.e., at the surface and $1 \mathrm{~mm}$ deep). Figure 2(b) shows the horizontal line intensity profile computed for each image for the $1 \mathrm{~mm}$ depth shown in Fig. 2(a). Figure 2(c) presents the spatial resolution analysis based on the measurement of the target diameter at various depths divided by the measured diameter at the surface. The fluorescent target diameter (Dia. [epth $x]_{\text {de }}$ ) was calculated based on twice the distance between the position of the maximum and the location where the intensity was 0.707 of the maximum using the line profiles [e.g., Fig. 2(b)]. The graph clearly shows the broadening of the fluorescent target as the depth increases, which was im- proved by implementation of the AFA. The focused lens system had the poorest spatial resolution at every depth tested. The lens-pinhole arrangement provided spatial resolution performance intermediate to the AFA and conventional lensbased systems. The image contrast as a function of depth was evaluated using the formula

$$
\text { Contrast ratio }=\frac{\operatorname{Average}\left(I_{\max 1}, I_{\max 2}\right)-I_{\min }}{\operatorname{Average}\left(I_{\max 1}, I_{\max 2}\right)+I_{\min }},
$$

where $I_{\max 1}$ and $I_{\max 2}$ represent the maximum light intensity of two fluorescent tubes $1.5 \mathrm{~mm}$ from each other, and $I_{\text {min }}$ represents the minimum light intensity between them, shown in Fig. 2(b). The contrast ratio was much higher for ADFI than the conventional and lens-pinhole approaches at all target depths tested [Fig. 2(d)]. Furthermore, the contrast ratio appeared to be relatively invariant up to a depth of $0.5 \mathrm{~mm}$ for ADFI, where with the other methods, the contrast ratio dropped by nearly twofold. These results demonstrated that ADFI had better overall imaging performance when compared to a conventional lens or lens and pinhole arrangement.

In order to demonstrate the capability of the ADFI at small animal imaging, a near-infrared (NIR) optical bone marker (IRDye 800-CW BoneTag) in a hairless mouse was used. This compound combined a fluorescent agent with a calciumchelator and served as a marker of the mineralization process. For this experiment, we used a SKH1 mouse (6 weeks old) and an intraperitoneal injection of $2 \mathrm{nmol}(100 \mu \mathrm{l})$ dye. The 


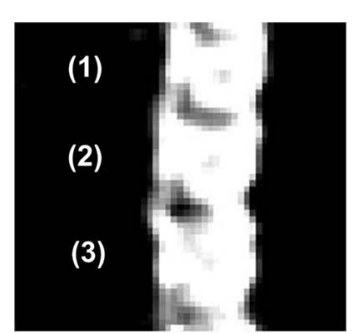

(a)

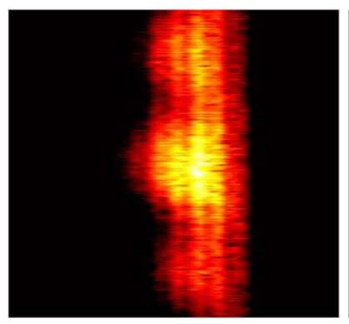

(c)
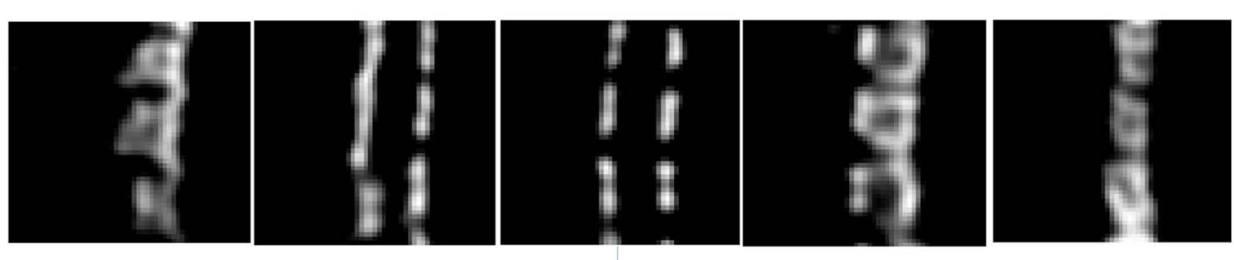

(b)

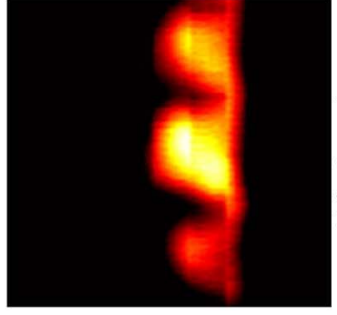

(d)

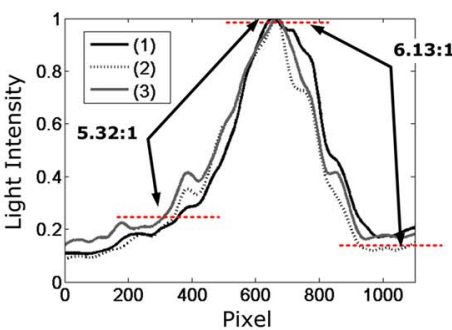

(e)

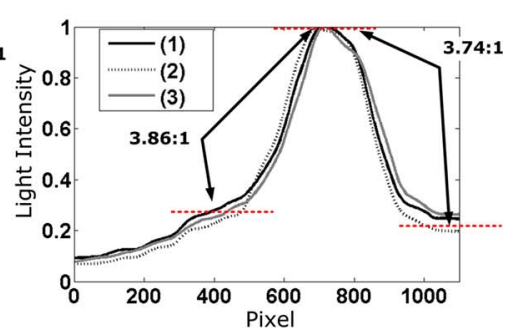

(f)

Fig. 3 Mouse ADFI bone imaging with comparison to x-ray CT and conventional reflectance-based imaging. (a) Maximum intensity projection of five x-ray CT slices; (b) three vertebrae of the spine of a hairless mouse. Images in (b) represent 0.625-mm-thick slices though the animal proceeding from shallowest (left) to deepest (right) in increments of $0.625 \mathrm{~mm}$. (c) Angular domain fluorescent image of three vertebrae of the mouse spine corresponding to (a) and (b). Camera integration time was $2 \mathrm{~s}$ for each line scan. (d) Conventional reflectance-based image of three vertebrae of the mouse spine indicated in (a) and (b). Each image (a) to (d) represents a field of view $1 \mathrm{~cm}$ wide by $1 \mathrm{~cm}$ high. (e) Fluorescence intensity line profiles corresponding to (c). (f) Fluorescence intensity line profiles corresponding (d).

mouse was euthanized $48 \mathrm{~h}$ later, and the spine was imaged. For imaging, the mouse was placed in a 5 -cm optical path cuvette in a prone position (i.e., back to the detector). We also obtained volumetric images from the same animal using a clinical x-ray computed tomography (CT) scanner (GE) for comparison. As shown in Fig. 3, vertebrae of the mouse spine were visible by x-ray CT [Figs. 3(a) and 3(b)], ADFI [Figs. $3(\mathrm{c})]$ and by conventional reflection-based fluorescence imaging [Fig. 3(d)]. The lens-based imaging system used the same optical setup (including pulsed laser and gated camera), but without the use of AFA and Keplerian lens system.

Analysis of the x-ray projection images of the horizontal diameter of the vertebra numbered 1 to 3 in Fig. 3(a) were $2.7 \mathrm{~mm}, 3.0 \mathrm{~mm}$, and $3.0 \mathrm{~mm}$, respectively. By a similar analysis of the corresponding vertebrae using the ADFI image [Fig. 3(c)], we obtained $2.8 \mathrm{~mm}, 3.2 \mathrm{~mm}$, and $2.5 \mathrm{~mm}$ in the same order. Similar measurements performed with conventional lens-based detection were $2.9 \mathrm{~mm}, 3.2 \mathrm{~mm}$, and $2.1 \mathrm{~mm}$ [Fig. 3(d)]. These measurements showed an error between ADFI and $\mathrm{x}$ ray of less than $17 \%$ for the third vertebrae [deepest, as shown in Fig. 3(b)], while the error between the lens-based measurement and $\mathrm{x}$ ray for the third vertebrae was $30 \%$. The measured light intensity of ADFI was more uniform compared to the surface-weighted conventional lens-based measurement. For all vertebrae, the background fluorescence intensity (measured 300 pixels to the left and right of the peak intensity) decreased by approximately 5.3 and 6.1 times to the left and right, respectively [shown in Fig. 3(e)]. Similar measurements performed using the lens-based imaging system without the AFA [Fig. 3(f)] resulted in the background fluorescence intensity decreasing by approximately 3.9 and 3.7 times to the left and right, respectively. Therefore, based on the worst case ADFI contrast measurements, there was at
$>30 \%$ improvement in contrast compared to corresponding contrast measurements made with the conventional lens-based imaging system.

In addition, ADFI appeared to have better sensitivity to fluorescent emission from deep within the animal compared to conventional lens-based imaging. Although conventional lensbased imaging resulted in more defined spatial boundaries of the fluorescent target, the resultant image was surfaceweighted (i.e., fluorescence originating close to the surface appeared brighter compared to deeper emissions). This effect was clearly observed for vertebra 3 , which was deeper in tissue when compared to the other two vertebrae [as shown in shallow x-ray images in Fig. 3(b)]. As expected, vertebra 3 appeared dimmer in the conventional reflection-based image [Fig. 3(d)]. However, in ADFI scan, the same vertebra was brighter and more representative of the true diameter as measured by x-ray CT imaging [Fig. 3(a)].

In summary, phantom and animal model tests demonstrated the usefulness of employing micromachined angular collimating array for fluorescent imaging to enhance the localization of fluorescently labeled targets. ADFI has several advantages over other fluorescence imaging modalities, including a larger field of view, higher image resolution, and better image contrast at tissue depths exceeding $1 \mathrm{~mm}$. This system could be used ultimately for the detection and sizing of tumor-targeted fluorescent agents at tissue depths significantly greater than conventional reflection-based imaging.

\section{Acknowledgments}

This project was funded by grants from the Natural Sciences and Engineering Research Council of Canada (NSERC) to Bozena Kaminska and Dr. Jeffery J. L. Carson. In addition, 
partial funding was provided to Fartash Vasefi by the Translational Breast Cancer Studentship from the London Regional Cancer Program.

\section{References}

1. R. Weissleder and V. Ntziachristos, "Shedding light onto live molecular targets," Nat. Med. 9, 123-128 (2003).

2. F. R. Wouters, P. J. Verveer, and P. I. H. Bastiaens, "Imaging biochemistry inside cells," Trends Cell Biol. 11, 203-211. (2001).

3. E. B. Brown, R. B. Campbell, Y. Tsuzuki, L. Xu, P. Carmeliet, D Fukumura, and R. K. Jain, "In vivo measurement of gene expression, angiogenesis and physiological function in tumors using multiphoton laser scanning microscopy," Nat. Med. 7, 864-868. (2001).

4. R. Hoffman, "Green fluorescent protein imaging of tumour growth, metastasis, and angiogenesis in mouse models," Lancet Oncol. 3(9), 546-556. (2002).

5. G. H. Chapman, M. Trinh, N. Pfeiffer, G. Chu, and D. Lee, "Angular domain imaging of objects within highly scattering media using sili- con micromachined collimating arrays," IEEE J. Sel. Top. Quantum Electron. 9, 257-266 (2003).

6. F. Vasefi, B. Kaminska, P. K. Y. Chan, and G. H. Chapman, "Multispectral angular domain optical imaging in biological tissues using diode laser sources," Opt. Express 16, 14456 (2008).

7. N. Pfeiffer, P. K. Y. Chan, G. H. Chapman, F. Vasefi, and B. Kaminska, "Optical imaging of structures within highly scattering material using a lens and aperture to form a spatiofrequency filter," Proc. SPIE 6854, 68541D (2008).

8. F. Vasefi, B. Kaminska, G. H. Chapman, and J. J. L. Carson, "Image contrast enhancement in angular domain optical imaging of turbid media," Opt. Express 16, 21492 (2008).

9. F. Vasefi, P. K. Y. Chan, B. Kaminska, G. H. Chapman, and N. Pfeiffer, "An Optical Imaging Technique Using Deep Illumination in the Angular Domain," IEEE J. Sel. Top. Quantum Electron. 13, 16101620 (2007)

10. F. Vasefi, B. S. Hung, B. Kaminska, G. H. Chapman, and J. J. L. Carson, "Angular domain optical imaging of turbid media using enhanced micro-tunnel filter arrays," Proc. SPIE 7369, 73691N (2009). 